\title{
Conocimiento que tiene el personal de salud del servicio de maternidad sobre lactancia materna exclusiva ${ }^{1}$
}

\author{
Beatriz Villalobos Núñez ${ }^{2}$
}

Institución: Hospital Max Peralta de Cartago. Costa Rica

\section{COMO CITAR}

Villalobos, B. (2011). Conocimiento que tiene el personal de salud del servicio de maternidad sobre lactancia materna exclusiva. [en línea].Rev. Enfermería Actual en Costa Rica, 21, 1-20 [citado (fecha)]. Disponible World Wide Web: <http://www.revenf.ucr.ac.cr/amamantamiento.pdf $>$ ISSN 1409-4568

\section{RESUMEN}

El siguiente artículo tiene como propósito presentar los resultados sobre el conocimiento que tiene el personal médico y de Enfermería que labora en el Servicio de Maternidad del Hospital Dr. Max Peralta de Cartago con la finalidad de fomentar prácticas que favorezcan el amamantamiento exclusivo y fortalecer la Clínica de Lactancia Materna de ese nosocomio. La población estuvo conformada por 28 personas, miembros del equipo de Enfermería y Medicina de la clínica de Lactancia Materna. Se diseñó un estudio cualitativo de tipo investigación acción. La información fue recolectada a través de la utilización de la entrevista. Dentro de los hallazgos más significativos se encontró que existen diferencias en el conocimiento que poseen los participantes en cuanto a la técnica de amamantamiento. Es necesario que la gestión del servicio de maternidad contemple los lineamientos del funcionamiento de la clínica de lactancia materna para fortalecer la promoción y mantenimiento de la misma.

Palabras clave: Enfermería, Lactancia-materna, Salud-infantil.

\footnotetext{
${ }^{1}$ Fecha de recepción: 3 agosto del 2011

Fecha de aceptación: 20 de setiembre del 2011

${ }^{2}$ Enfermera. Profesora Instructora de la Escuela de Enfermería de la Universidad de Costa Rica. Correo electrónico: bvlleaenf@yahoo.es
} 


\title{
Knowledge that have the health staff of the maternity service on exclusive breastfeeding ${ }^{3}$
}

Beatriz Villalobos Núñez ${ }^{4}$

Institution: Hospital Dr. Max Peralta de Cartago. Costa Rica

\section{Cited}

Villalobos, B. (2011). Knowledge that have the health staff of the maternity service on exclusive breastfeeding. [on line]. Rev. Enfermería Actual en Costa Rica, 21, 1-21 [cited (date)]. Available World Wide Web: <http://www.revenf.ucr.ac.cr/amamantamiento.pdf $>$ ISSN 1409-4568

\begin{abstract}
The following article presents the results of educational needs assessment aimed at medical and nursing staff to strengthen Breastfeeding Clinic Hospital Max Peralta, the purpose of this was to identify the educational needs of staff regarding breastfeeding, to promote practices that promote exclusive breastfeeding. The same was done in 2005. By using a qualitative action research type. The population consisted of 28 people team members professional and technical nursing and two medical professionals. Information was collected through a structured interview. Among the significant findings found the absence of a physical and organizational structure of the breastfeeding clinic. On the other hand there are differences in the knowledge possessed by the participants regarding the breastfeeding technique. It is necessary that the maternity service management guidelines provide for the operation of the breastfeeding clinic to strengthen the promotion and maintenance of it.
\end{abstract}

Key words: Child-health, Exclusive-breastfeeding, Nursing.

\footnotetext{
${ }^{3}$ Date of receipt: August 3, 2011

Date of acceptance: September 20, 2011

${ }^{4}$ Enfermera. Profesora Instructora de la Escuela de Enfermería de la Universidad de Costa Rica. Correo electrónico: bvlleaenf@yahoo.es
} 


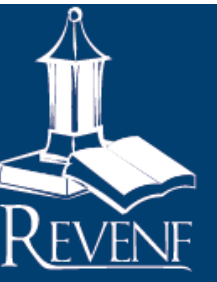

\section{INTRODUCCIÓN}

La lactancia materna es considerada por los especialistas en la materia como la única forma de satisfacer todas las necesidades nutritivas de los niños y las niñas menores de seis meses. Es la forma natural de alimentación que tienen las personas al nacer, por lo tanto esta depende de la actitud y capacidad de la madre y las condiciones ambientales y sociales que favorezcan esta práctica (Cerezo, 1995; Cooney, 1994; Ministerio de Salud de Costa Rica, 1986). Es a través de la alimentación exclusiva con leche materna como se logran disminuir los riesgos de enfermedades infecciosas y alergias en los primeros doce meses de vida, además de que se reducen las posibilidades de tener en el futuro personas con padecimientos tales como diabetes, obesidad y otras enfermedades crónicas. (Burgess, 2006)

Además de todos estos beneficios físicos brindados por la lache materna al infante, existen también otros en el ámbito social, familiar, económico y ecológico. (Helsing, 1983; OMS, 1990; Savage,1989) . Dentro de estos beneficios, a parte del nutricional, que recibe el niño o la niña es muy importante lo referente al apego materno, (Chaves \& Morice, 1998) al fortalecimiento del vínculo madre hijo que se da mediante las prácticas de alimentación natural con leche materna.

A pesar de estas ventajas, a partir de 1950 se empezó a experimentar en nuestro país, y el resto del mundo, una tendencia al destete precoz y la introducción de alimentos sólidos de forma temprana (Vargas \& López, 1980). Es a partir de los setenta que, a raíz de esta situación, se gesta un movimiento a favor de la promoción de la lactancia materna y protección de la misma. (Aguirre, E. 2007)

Este movimiento produce, a nivel nacional, una serie de actividades (discusión, seminarios, entre otras) en las diferentes instituciones que en ese momento tenían relación con los temas de salud, con el fin de implementar estrategias que contribuyeran a proteger la alimentación de los niños y niñas menores de seis meses con leche materna. A raíz de todo esto surge la creación de la Comisión de Lactancia Materna en Costa Rica, la cual, en conjunto con el Ministerio de Salud y todas las legislaciones nacionales que tengan que ver con la lactancia materna, asume la tarea de velar por su promoción y protección. (Ministerio de Salud, 2009)

El esfuerzo realizado en la promoción de la lactancia materna es fruto de esta comisión, y como resultado de esto surgen las clínicas de lactancia materna en las diferentes maternidades del país. Para el año 2005 en Costa Rica se contaba con nueve clínicas de lactancia materna ya establecidas (Aguirre, 2007; Jiménez, 2004). Sin embargo, existen servicios de maternidad que en la fecha citada no contaban con una.

En la encuesta nacional de hogares del 2006, los datos obtenidos reflejaron que tan solo el $18.7 \%$ de los niños y niñas eran amamantados exclusivamente con leche materna hasta la edad de seis meses, información alarmante si se compara a Costa Rica con otros países del mundo, como es el caso de Chile con un 63\% y Guatemala con un 51\%. Si bien los datos para Costa Rica, comparando el 2000 con el 2006, son favorables, aun estamos muy por debajo de otros países. (Cháves, Mata \& Moya, 2007)

A raíz de todo lo anterior, el sistema de salud nacional ha realizado esfuerzos valiosos en la promoción de estrategias para el fomento de la lactancia natural, rescatándose acciones como la capacitación del personal de salud; la educación a nivel de la enseñanza general básica en las escuelas formadoras de recurso humano en 


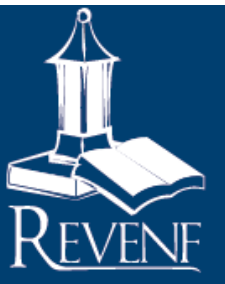

salud; la educación a la población costarricense en general; la generación de la legislación que protege a la madre trabajadora y a su hijo (a); el desarrollo de un control de la comercialización de sucedáneos de la leche materna y finalmente el desarrollo de proyectos de investigación que brinden información objetiva y oportuna para la adecuada implementación de políticas y actividades de promoción de la lactancia natural que son llevadas a cabo en el país. (Ministerio de Salud, 2009)

Como parte de estos esfuerzos, el presente artículo muestra los resultados parciales de una investigación que pretendió cimentar y afianzar el rol del profesional en Enfermería en el área de fomento de la salud, promoviendo prácticas de lactancia materna, con el fin de ayudar a detectar, en forma temprana, factores de riesgo presentes y que puedan interferir de algún nodo en el proceso de amamantamiento, afectando así la salud de la madre y su bebé.

Para la sociedad costarricense la presencia de este tipo de investigaciones es de suma relevancia, ya que las prácticas de lactancia materna tienen una relación directa con la nutrición infantil y la reducción de la misma. Además esta práctica va aunada a la disminución de enfermedades causantes de grandes problemas de salud infantil a lo largo de la historia, como lo han sido las diarreas. Asociado a esto, para la población nacional contar con profesionales de Enfermería capacitados en el tema de la lactancia, no solo teóricamente sino también implementando prácticas que favorezcan el amamantamiento exclusivo, es un gran aporte, dado que la Enfermería es una de las disciplinas de la salud permanentemente en contacto con las personas, guiándolas en la construcción de su salud.

Este estudio permitió reconocer diferentes vacíos de conocimiento, los cuales son de gran utilidad para la disciplina de Enfermería, entre ellos el correcto funcionamiento de una clínica de lactancia materna y la importancia de la participación del profesional de Enfermería en lo referente al fomento y promoción de la lactancia natural, contando para ello con el apoyo tanto del profesional de salud como del grupo social de convivencia.

Este artículo tiene como objetivo exponer los resultados de un diagnóstico sobre conocimientos del personal médico y de Enfermería sobre lactancia materna exclusiva que labora en el servicio de maternidad del Hospital Dr. Max Peralta de Cartago con la finalidad de fortalecer La Clínica de Lactancia Materna de ese nosocomio.

\section{MATERIALES Y METODOS}

La investigación se contempló dentro de un paradigma naturalista, con un enfoque cualitativo de tipo investigación acción, en la cual, tanto el investigador como el sujeto investigado, buscan una solución conjunta al problema o necesidad identificada en el proceso investigativo (Barrantes, 2010). En el estudio se realizó un diagnóstico de necesidades educativas para fortalecer los procesos que se gestan en la Clínica de Lactancia Materna del Hospital Dr. Max Peralta de Cartago, con el fin de fomentar la práctica de la lactancia materna exclusiva. 


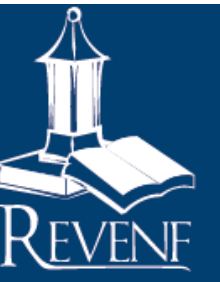

La población del estudio estuvo constituida por un total de 30 participantes, de los cuales 28 pertenecían al personal de Enfermería, incluidos tanto profesionales como técnicos, y 2 pertenecían al personal médico. La totalidad de los participantes laboraban en el servicio de maternidad del Hospital Dr. Max Peralta.

La recolección de la información se obtuvo mediante la utilización de la técnica de entrevista, para lo cual se formuló una guía de entrevista con preguntas cerradas y abiertas, las respuestas de las mismas fueron transcriptas tal y como las expresó el sujeto. Esta encuesta les fue aplicada al personal médico y de Enfermería en el servicio de maternidad con el fin de realizar un diagnóstico sobre los conocimiento en lactancia materna exclusiva, y con base en éste, crear la propuesta educativa para el fortalecimiento de la Clínica de Lactancia Materna. La entrevista fue aplicada por la investigadora, quien permaneció con los sujetos individualmente mientras contestaban, y al mismo tiempo realizaba las anotaciones pertinentes.

La categoría de análisis "conocimiento" se desglosó en las siguientes subcategorías: lactancia materna exclusiva, técnica de amamantamiento, preparación para amamantar, acompañamiento y manejo de problemas tempranos. Esto permitió organizar y transcribir la información tal y como acontece según los sujetos participantes.

Los datos recolectados por medio de los instrumentos aplicados (entrevista semiestructurada, observación no participante) se analizaron utilizando la triangulación de datos (Barrantes, 2010), en este caso provenientes de diferentes fuentes, lo cual permitió realizar un análisis más objetivo y cercano a la realidad, para finalmente realizar una síntesis en la que fueron identificados los aspectos más críticos del diagnóstico.

\section{Consideraciones éticas}

En el desarrollo de este estudio se trabajó directamente con personas, por lo tanto una parte imprescindible por considerar fueron los principios de la bioética, descrita por Serrano (2003) de la siguiente manera "la ética de todas las intervenciones sobre las personas, y por tanto, la salud pública, la promoción de la salud y la educación para la salud, que son intervenciones sobre el hombre, tienen una gran importancia..." (p.592)", de esta manera, al trabajar con personas, se debe de respetar los principios de bioética para asegurar su integridad y conservación de derechos.

Basado en lo anterior se consideran los siguientes principios de bioética, tomados de Blázquez (2004): Autonomía: significa respetar a las personas como individuos libres, teniendo en cuenta sus decisiones como fruto de sus valores. Este principio se hizo manifiesto al brindarle la oportunidad a las personas de participar libres y sin presiones, al mismo tiempo que se les informó de la libertad de retirarse o negarse a participar en el momento que lo consideraran. Además, se les facilitó toda la información referente a los objetivos y manejo de la información obtenida. Los y las participantes firmaron, en el documento de la entrevista, su anuencia a participar en la investigación de una forma libre y voluntaria. Veracidad: considerado como el principio de no mentir, se hace efectivo al ofrecerles a las personas toda la información tal cual era presentaba sin ocultar ni negar la misma, de modo que los participantes recibieron la información de una forma transparente, sin ninguna alteración. Privacidad: tiene como objetivo no permitir la divulgación de información confidencial de las personas que, directa o indirectamente, se obtenga sobre su salud o estilo de vida. El anonimato de las personas fue mantenido para respetar este principio. 


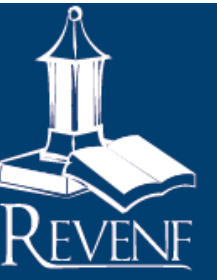

Beneficencia y no maleficencia: busca el beneficio y el no hacer daño a las personas. El respeto a este principio se consiguió mediante actividades que no fueran en contra los valores de las personas participantes. Además, se llevo a cabo una sesión con el personal para devolver de los resultados, con el propósito de que los pudieran utilizar para su propio beneficio.

Finalmente es indispensable conocer que, con la intensión de dar mayor validez a la investigación y cumpliendo con los criterios de rigor metodológico, la información recolectada fue transcrita tal cual la presentaban los sujetos, y de esta manera se pudiera reflejar mejor la realidad que se vive en el contexto estudiado, además para confirmar dicha información se realizaron observaciones no participantes y posteriormente se le presentaron los resultados obtenidos al personal de enfermería del servicio de maternidad y en conjunto con las personas encargadas directas de lo referente a la clínica de lactancia materna se definió según los resultados una propuesta educativa.

\section{RESULTADOS}

Los resultados se presentarán según la categoría denominada conocimiento, de la cual se desprenden las subcategorías de análisis evidenciadas en los resultados. La primera de ellas correspondió al conocimiento sobre la lactancia materna exclusiva, encontrándose que, según las respuestas obtenidas, todos los encuestados hacen referencia a la lactancia exclusiva, demostrando que poseen conocimiento de este concepto. Sin embargo, uno de los aspectos rescatables, es la opinión manifestada de que la lactancia exclusiva es "cosa de la madre".

Con respecto al conocimiento sobre técnica de amamantamiento, se encontraron respuestas como: "Colocarse en una posición cómoda, depende de la madre la posición que más le convenga y acomodarse al bebé para que pueda alcanzar el pecho" (P1), "Existen diferentes: sentada con el bebé, o apoyada en una almohada acostada apoyando al bebé en su brazo, le introduce toda la areola y pezón en la boca"(P15), "Posición cómoda"(P19), "Una buena posición que el bebé tenga en su boca todo el pezón"(P20).

En cuanto al aspecto de apego materno, queda demostrado que los participantes poseen un criterio amplio con respecto al tema, y hacen una estrecha relación entre el amamantamiento y el apego madre hijo o hija. La respuesta siguiente es una muestra de esto: "Amamantar influye grandemente porque al sentir caricias, amor, diálogo, y apego a su hijo existe más amor.” (P22).

Uno de los aspectos de gran importancia para este estudio, fue conocer acerca de cómo funciona la clínica de lactancia materna. Dentro de los resultados se encontró lo siguiente: "Más o menos no funciona muy bien" (P4), "Aquí no hay pero la finalidad es que la madre pueda sacarse la leche y lactar" (P5), "Si, aunque en este hospital aun no funciona como debe de ser" (P12). Estos comentarios reflejan el inadecuado funcionamiento de este programa, también denota el desconocimiento, por parte del personal, con respecto de lo qué es la clínica y cuál es su funcionamiento en la institución. Además, cuatro de los encuestados refirieron no saber cuál es el funcionamiento de la clínica de lactancia materna.

En cuanto a los programas con los que cuenta el personal del servicio de maternidad para fomentar el amamantamiento, las respuestas no coinciden entre sí, lo que demuestra una deficiencia en este aspecto, comparado con lo que evidencia la observación a las madres, pues si bien es cierto se les pregunta si su bebé está 


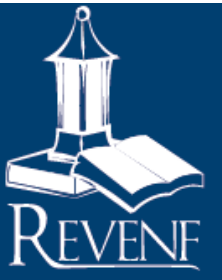

mamando, no se les enseña como hacerlo adecuadamente, excepto por una situación en donde la enfermera ayudaba a la madre.

Se indagó sobre el conocimiento que posee el personal encuestado sobre las posibilidades de amamantamiento en caso de situaciones en que los infantes presenten condiciones como labio leporino o paladar hendido. Se evidenció que todos coinciden que estas malformaciones no son un impedimento para el inicio de la lactancia.

Continuando con el análisis de las subcategorías, un aspecto fundamental analizado, desde el conocimiento, es la producción de leche materna. Al investigar sobre los aspectos que pueden llegar a inhibir la producción de leche, las respuestas repetidas con más frecuencia son el estado emocional y de estrés en la madre. Asimismo, algunas respuestas hacen referencia a una causa común de baja producción de leche: la falta de estímulo o poca succión por parte del niño(a).

Por otra parte se indagó acerca del conocimiento que poseen los sujetos encuestados sobre las situaciones en las cuales se debe suspender la lactancia materna, todas las respuestas se ubican en situaciones como: "Enfermedad de la madre y embarazo" (P1), "SIDA" (P2), "No se suspende" (P3).

Con respecto a los aspectos mecánicos del amamantamiento, se preguntó sobre el conocimiento que poseían los participantes acerca de la posición correcta para el proceso de amamantado. El personal entrevistado destacó la comodidad de la madre y el niño(a) como un factor de gran importancia para este proceso, por lo que la madre debe mantener una posición cómoda para ambos.

Al comparar las respuestas concernientes a posición correcta para el amamantamiento, con las respuestas obtenidas en técnica de amamantamiento, donde se evaluó sobre los conocimientos, es posible identificar como el personal tiende a confundir ambos temas.

Con respecto al conocimiento sobre por cuánto tiempo debe amantarse al bebé, 13 participantes mencionaron que es el niño(a) quien debe decidir si ya esta satisfecho. Por otro lado, en 11 respuestas se señaló que la madre, al recibir una educación previa que le permita reconocer cuando el niño(a) ha recibido suficiente leche, puede finalizar la amamantada sin privar al niño de su alimento.

Referente a la temática de la relación entre oferta y demanda, y su importancia en la lactancia materna, la respuesta del personal evidencia una confusión entre estos aspectos y el aumento de la producción de leche relacionado con la succión.

\section{DISCUSION}

La población entrevistada hizo referencia a un concepto claro de lactancia materna exclusiva (Asociación Española de Pediatría, 2004; OMS, 1993), sin embargo es importante mencionar como la madre es considerada como la única de la que depende este proceso. Pero es claro que muchas madres solas difícilmente lograrán llevar a cabo una lactancia exclusiva exitosa (OMS, 1993), y es aquí donde la clínica de lactancia debe hacer un arduo 


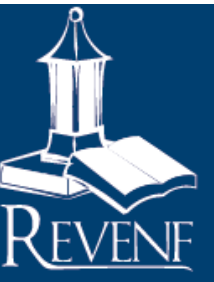

Revista Electrónica

Enfermería Actual en Costa Rica

esfuerzo para involucrar, en el proceso de amamantamiento, a otras personas dentro del núcleo social de convivencia, con el fin de que puedan apoyar a la madre.

En relación con la técnica de amamantamiento, se encontró una confusión entre técnica de amamantamiento y posiciones para amamantar, a pesar de que muchas personas tratan de explicar la técnica, en su intento mezclan los dos conceptos, lo cual demuestra una debilidad en el personal a la hora de ayudar a las madres, pues la madre puede sentirse cómoda pero no necesariamente está teniendo un proceso de amamantamiento ideal, debido a que una incorrecta succión puede afectar directamente la producción y extracción de la leche (González, 2009), siendo esto un factor de riesgo para que la madre abandone la lactancia materna (OMS, 1993).

El hecho de que una madre desconozca la técnica adecuada para colocar su hijo o hija, va a favorecer que los pezones de la madre se lesionen debido a la succión insistente y fuerte por parte del infante por intentar obtener la leche (Asociación Española de Pediatría, 2004). Es en estos aspectos donde se revela la importancia de que el personal de salud tenga claridad en cuanto al concepto de la técnica para amamantar para orientar adecuadamente a la madre, lo que hace más que necesario retomar la educación continua dirigida al personal de Enfermería en cuanto a lactancia materna.

En relación del apego materno, Cháves y Morice (1998) dicen que “... favorece los vínculos afectivos entre la madre y su hijo (a) y establece una relación emocional que promueve un óptimo desarrollo en el niño (a.)" (p. 5). De acuerdo con la opinión de los participantes en el estudio, el apego es la relación afectiva, de seguridad, amor y hasta de protección establecida entre madre e hijo (a), y esta relación es favorecida por el acto de amamantar, el cual lo conciben como un acto de amor. Por lo tanto se debe propiciar un ambiente adecuado para favorecer esta relación. Sin embargo, se debe tomar en cuenta que en muchas ocasiones la apatía hacia algo surge por el desconocimiento y no propiamente por realizar determinada práctica. De tal manera que si una mujer madre no sabe cómo alimentar a su hijo o hija será necesario que el personal de salud la acompañe en el proceso.

Lo anterior es posible lograrlo favoreciendo prácticas como colocar al bebé, inmediatamente después de su nacimiento, en el pecho de la madre y permitirle succionar, identificar si la madre o el bebé tienen alguna dificultad para corregirla tempranamente, lo que puede significar que en adelante la relación sea mejor, beneficiando el amamantamiento y al mismo tiempo, el vínculo madre hijo o hija. Cháves y Morice (1998), plantean que existe un periodo crítico para el establecimiento del vínculo afectivo entre la madre y el niño (a), es el que comprende la primera hora posterior al nacimiento.

Respecto del funcionamiento que debería tener una clínica de lactancia materna. (Caja Costarricense de Seguro Social \& Ministerio de Salud, 1994), se manifestaron diferentes opiniones entre el personal de salud participante. Se destacaron elementos importantes como el velar a un cien por ciento por el adecuado proceso de lactancia natural, debe dársele apoyo a la madre, al niño (a) y el núcleo social de convivencia, ofreciéndole la ayuda necesaria para llevar a cabo una lactancia natural exitosa, elementos que coinciden con el correcto funcionamiento de la clínica de lactancia materna.

Cabe rescatar como en la actualidad el parto intrahospitalario es cada vez más frecuente (INEC, 2000), por lo tanto la madre inicia el amamantamiento en el hospital, donde debe recibir la primera orientación y ayuda para 


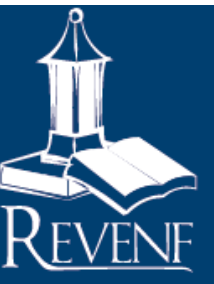

que pueda tener un exitoso inicio de la lactancia natural, de manera que la calidad de la educación y ayuda recibida por la madre puede beneficiar el proceso de amamantamiento o bien, si esta es inadecuada, perjudicarlo. Por esta razón el equipo de salud debe mantenerse actualizado y trabajar en conjunto con unificación de criterios, que les permita ir en busca de un fin común: la lactancia exitosa (Asociación Española de Pediatría, 2004).

Es importante señalar que el personal de salud del servicio de maternidad tiene amplia comprensión de que el amamantamiento a bebés con labio leporino o paladar hendido, aunque es más difícil, no es imposible. Mencionan que todo es cuestión de buscar la manera más indicada y darle tiempo al niño(a) para aprender a succionar y darle apoyo a la madre, tanto por parte de la familia como del personal de salud. (Asociación Española de Pediatría, 2004).

Con respecto de la producción de leche, los participantes dicen que esto se debe a aspectos emocionales, los cuales pueden inhibir la producción de leche. Según (Savage, 1989) no solamente los aspectos emocionales intervienen en la producción sino también en la cantidad de veces que el bebé succiona y la introducción de otro tipo de alimentación, por lo tanto queda claro que la madre necesita instrucción sobre este aspecto de parte del profesional de salud. (OMS, 1993)

La enfermedad de la madre en el periodo de lactancia, es otro factor que ha intervenido en una lactancia exitosa. No obstante, la literatura especializada dice que la enfermedad de la madre no es un factor para dejar de amamantar al bebé (Asociación Española de Pediatría, 2004), sin embargo, existen ciertas recomendaciones en casos muy especiales, por ejemplo, los resultados hacen referencia a enfermedades como el VIH/SIDA como un factor para suspender el amamantamiento, sin embargo la UNICEF (2009) plantea grandes cambios en este aspecto, en donde si las madres y los niños reciben los antirretrovirales tempranamente la lactancia materna no se ve afectada sino más bien favorecida.

Los entrevistados plantean también la gestación de la madre como un factor para interrumpir la lactancia materna. En cuanto al amamantamiento y un nuevo embarazo, médicamente no está contraindicado, siempre y cuando no hallan otros factores de riesgo. Sin embargo, muchas madres se asustan y creen que le puede pasar algo ya sea al lactante o al gestante. En estos casos es importante la valoración de la edad del niño(a) amamantado, pues puede que ya tenga la edad suficiente para ser destetado. Debe considerar además la nutrición de la madre, la cual debe de ser muy buena para no perjudicar el bienestar de ninguno.

En los resultados se encontró una opinión dividida en cuanto a quién debe de finalizar la amamantada, si la madre o el niño o niña. La bibliografía consultada menciona que no debe ponérsele una duración determinada a las amamantadas, ya que el niño(a) en general se duerme o suelta el pecho cuando está satisfecho. Y los niños(as) deben terminar espontáneamente la alimentación (Asociación Española de Pediatría, 2004). En estos casos es importante que la madre sepa valorar si su hijo(a) realmente se ha alimentado, si ha succionado el tiempo necesario, y ha bajado leche. Esto la madre lo puede aprender, solo es cuestión de motivarla a escuchar, por así decirlo, el lenguaje de la lactancia natural, y recordar que todo niño(a) es diferente uno del otro, no se puede pretender tener comportamientos iguales.

Unido a la temática anterior, se encuentra la relación entre la oferta y la demanda y su importancia en la lactancia materna. La respuesta del personal evidencia una confusión entre oferta y demanda y el aumento de la producción 


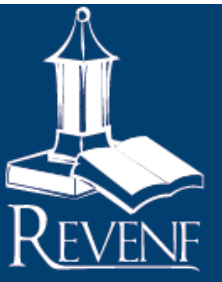

relacionado con la succión, si bien es cierto, en la medida que se respete la ley de oferta y demanda, la producción aumentará, no es lo mismo, pues la oferta y demanda se centra básicamente en no ponerle horarios fijos a los bebés sino más bien que sean ellos mismos los que decidan cuando comer según sus necesidades (Asociación Española de Pediatría, 2004; Wellstart International, 1994).

En general el personal muestra conocimiento en el tema de la lactancia natural, sin embargo se presenta incongruencias, o diferencias en algunos aspectos de gran relevancia tanto para el funcionamiento de una clínica de lactancia como para el beneficio de las usuarias del servicio de maternidad.

\section{CONCLUSION}

Es necesario fortalecer el servicio de maternidad con material que le permita al personal implementar mayor cantidad de estrategias de promoción del amamantamiento exclusivo, las cuales involucren tanto a la madre como a su núcleo social de convivencia, de tal manera que al egresar del hospital pueda contar con una red de apoyo.

La gerencia del servicio de maternidad debe de retomar las políticas y normas para el establecimiento de una clínica de lactancia materna, para lo cual es importante tomar en cuenta todos sus elementos, de tal manera que puedan actualizar los conocimientos del personal de salud y disponer permanentemente de presupuesto y de una persona dedicada a la gestión de las actividades indispensables tanto para el adecuado funcionamiento de la clínica como para que el trato y apoyo brindado a las usuarias y usuarios contribuya al fomento de la lactancia natural exclusiva hasta los seis meses y a la lactancia materna prolongada.

\section{REFERENCIAS}

Aguirre, E. (2007). Propuesta para la creación de la Clínica de lactancia materna en la Universidad de Costa Rica, BINASSS.

Asociación Española de Pediatría. (2004). Lactancia Materna: guía para profesionales. Madrid, España. Recuperado el: 2607-2011. De: http://www.nutrinfo.com/archivos/ebooks/lactancia_materna.pdf

Barrantes, R. (2010). Investigación. Un camino al conocimiento. Un enfoque cuantitativo y cualitativo. San José: EUNED.

Blazquez, N. (2004). La Bioética y los Hijos del Futuro. Madrid, España: Visión NET.

Burgess, A, (2006). Guía de nutrición de la familia. FAO. FIAT. PANIS.

Cerezo, R (1995) Manejo de las posibles dificultades de lactancia materna. Guatemala INCAP

Chaves, S., Mata, A. y Moya, R. (2007) Caja Costarricense de Seguro Social. Equipo Técnico Salud del Niño y la Niña. Resumen ejecutivo: Situación en Costa Rica de lactancia materna, según encuesta nacional de hogares y propósitos múltiples. INEC-2006. San José, Costa Rica. 


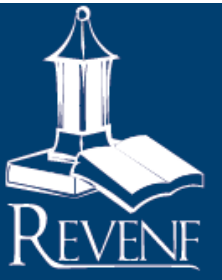

Chaves, S y Morice, A. (1998) La Promoción de la Lactancia Materna en Costa Rica. San José, Costa Rica.

Cooney, K y Labbok, M. (1994). La lactancia como una cuestión de la mujer: la salud, la planificación, el trabajo y el feminismo. s.e. Suplemento especial del International Journal of Ginecology and Obstetrics. N 47.

González, C, (2009) Un regalo para toda la vida. Guía de lactancia materna. Ediciones Planeta Madrid, S.A. España.

Helsing, E y Savage, F. (1983). Guía práctica para una buena lactancia. Editorial Pax- México, México.

INEC, (2000). Estadísticas Vitales 2000: población, nacimientos, defunciones, matrimonios. Costa Rica.

Jiménez, C., (2003) Seminario de Graduación: fortalecimiento de la clínica de lactancia materna en el Hospital México para la prevención de mortalidad infantil. San José, Costa Rica.

Ministerio De Salud. Comisión Nacional de Lactancia Materna. (2009). Política Pública de Lactancia Materna. Recuperado el: 20-07-2011. De: http://www.ministeriodesalud.go.cr/gestores_en_salud/lactancia/politica_lactancia_materna.pdf

Ministerio de Salud y C.C.S.S. (1994). Normas de atención al recién nacido sano. Costa Rica. San José, Costa Rica.

Ministerio de Salud. (1986). Fomento del hábito de lactar al pecho. Un compromiso del personal de salud., s. e. San José, Costa Rica. Cuarta edición.

Morice, A y Chaves, S. (1998). La Promoción de la Lactancia Materna en Costa Rica. San José, Costa Rica.

OMS. (1993). Consejería en Lactancia Materna: curso de capacitación Recuperado el: 26-07-2011. De http://whqlibdoc.who.int/hq/1993/WHO_CDR_93.3_spa.pdf

OMS. (1990). Educación en lactancia materna para profesionales de la salud. Washington D.C.

Savage, F. (1989). Como ayudar a madres a amamantar. UNICEF, Guatemala.

Serrano, M. (2003). La educación para la salud del siglo XXI: Comunicación y Salud. Segunda Edición. Madrid, España: Díaz de Santos.

UNICEF, (2009). La infancia y el SIDA: cuarto inventario de la situación, 2009. www.unicef.org/publications.

Wellstart International y University of California San Diego (1994). Curriculum para el Manejo de la Lactancia: una guía para el personal docente de las escuelas de medicina, enfermería y nutrición. Primera Edición en español. San Diego, CA: Wellstart International. 